\title{
Disasters and climate change in the Pacific: Adaptive capacity of humanitarian response organisations
}

\begin{abstract}
Climate change is likely to affect the pattern of disasters in the Pacific and by extension, the organisations and systems involved in disaster response. This research focused on how immediate humanitarian health-related needs following disasters are met using the concept of adaptive capacity to investigate the resilience of organisations and the robustness of the broader system of disaster response. Four case study countries (Cook Islands, Fiji, Samoa, and Vanuatu) were chosen for deeper investigation of the range of issues present in the Pacific.

Key findings were that adaptive capacity was enhanced by strong informal communication and relationships as well as formal relationships, appropriate participation of traditional leaders and churches, and recognition and support for the critical role national disaster management offices play in disaster coordination. Adaptive capacity was found to be constrained by lack of clear policies for requesting international assistance, lack of coordinated disaster assessments, and limited human resources for health in disaster response. Limitations in psychosocial support and Australian medical services to meet specific needs were observed. Finally, the research revealed that both Pacific and Australian disaster response agencies would benefit from a strengthened 'future' focus to better plan for uncertainty and changing risks.
\end{abstract}

Key words: Pacific, disaster, climate change, adaptive capacity, humanitarian needs, health 


\section{Introduction}

A growing body of evidence links anthropogenic global warming to increased number of observed extreme weather events, with "plausible" evidence for a link to an increased severe storm potential (Coumou and Rahmstorf, 2012). Projections for tropical cyclone frequency for the Pacific follow global trends - i.e. less frequent tropical cyclones by the end of the $21^{\text {st }}$ century, however, there is an expected change in the relative proportion of severe storms (Knutson et al., 2010). Some countries will experience a greater number of severe storms, while for others, the proportion of severe storms will decrease.

The intersect between climate change, disasters, health and development is an area of concern for the development community (Bowen et al., 2012; Jones et al., 2010; World Bank, 2010). One of the key priorities identified by regional health stakeholders is to better understand how climate change will impact on health system's ${ }^{1}$ response to emergencies and disasters (APEDNN, 2010). Australia's development policy makers and practitioners also have an interest in better understanding how climate change may affect disaster response. Australia's development assistance to the Pacific aims to address immediate development needs, and increasingly, to support future needs which are influenced by climate change. Australian Agency for International Development's (AusAID's) 2011 Humanitarian Action Policy states Australia's commitment to respond effectively to disasters occurring simultaneously (increasingly possible under climate change scenarios), and to ensure the response is timely and coordinated (AusAID, 2011).

The purpose of this research was to assist the development of adaptive capacity for effective disaster response in the face of climate change, and thereby contribute to reducing vulnerability to climate driven disasters. The research focused on disasters, human health and climate change in the Pacific. Current gaps in human, technical and financial capacity in the Pacific region have the potential to increase with the added burden of climate change. Hence the aim was to provide insight into the resources, policies and systems needed in coming years to enhance adaptive capacity from both the Australian and Pacific perspectives, given the inherent uncertainty presented by climate change.

'Adaptive capacity' describes the ability of a system to adjust to climate change (including climate variability and extremes), to moderate potential damages, to take advantage of opportunities, or to cope with the consequences (IPCC, 2001). The research investigated adaptive capacity from two perspectives: 1) the adaptive capacity of Pacific island country (PIC) disaster response organisations, in terms of internal coordination, participation in national decision making, resource capacity, and how external assistance is requested and received; 2) the adaptive capacity of Australian disaster response organisations to support effective response. The research also provides analysis of how disaster assistance provided by Australian organisations supports or constrains the adaptive capacity of PICs.

The research team drew on literature across relevant disciplines to define a range of key determinants of adaptive capacity to support exploration of the concept (see Section 2: Methodology). Three research questions were developed to guide this study:

1. What constitutes the 'disaster response system' (DRS) for the immediate humanitarian needs post-disaster (health care, water and sanitation, psychosocial needs and food and nutrition) in Australia and each of the four case study PICs?

2. How do the inter-organisational determinants serve to strengthen or reduce adaptive capacity of the 'DRS'?

3. Which objective and subjective determinants are most significant in influencing the adaptive capacity of the organisations within the 'DRS'? 
The research focused on adaptive capacity associated with responding to some of the immediate health-related needs post disaster (identified in Question One) to allow specific and relevant recommendations to be developed. The relevance of additional immediate humanitarian needs following a disaster (e.g. shelter, protection, and logistics), is recognised, however the focus on our selection of health related needs after a disaster allowed a more in-depth assessment.

This paper provides Theory and Methods in Section 2, followed by Results and Discussion (both from the Australian and Pacific perspectives) in Section 3, and a Conclusion in Section 4.

\section{Theory and Methods}

A qualitative research methodology was used, which prioritised stakeholder participation and end-user engagement. Methods included desktop reviews, individual and group interviews, and in-country workshops. The research process was guided by a Project Reference Group (PRG, comprised of Pacific and Australian academics and practitioners) as a form of structured stakeholder engagement.

A Conceptual Framework (Figure 1) was developed to provide the scope for this study. The Conceptual Framework describes a cycle of adaptive learning within which the adaptive capacity of the DRS is influenced by a range of key determinants (Ekstrom et al., 2012). The DRS is defined, in the scope of this research, to be comprised of actors and agents from government and non-government sectors, governance structures (policies, plans and legislation) and the formal and informal networks that support them. Following from studies including Engle (2011) and Walker et al. (2004), the concept of adaptive capacity was used to assess the resilience of individual organisations and the robustness of the broader system of response. The DRS at the national scale was the focus of the research, with the institutional architecture of most interest, rather than the specifics of community based response mechanisms.

\section{Figure 1: Conceptual framework concerning adaptive capacity of the DRS}

Specific determinants of adaptive capacity were used to assess the DRS, and were drawn from literature that spans Earth System Governance (Biermann, 2007), climate change adaptation (Ekstrom et al., 2012), health resources (WHO, 2006; WHO, 2011; Bowen et al., 2012), resilience in institutions (McManus et al., 2008) and practice theory (Strengers, 2010).

Key determinants were categorised as inter- or intra-organisational; and objective or subjective. The key determinants of adaptive capacity were an important element of the Conceptual Framework and lie at the centre of the research focus. These are described in Box 1.

\section{Key determinants of adaptive capacity}

Inter-organisational level:

Architecture: The architecture involves the systems and institutions associated with disaster response across both national and international scales (Biermann, 2007).

Agency: Drawing on concepts such as power, control and decision making, agency goes beyond intergovernmental co-operation and requires an understanding of the partnership between 'whole of government' and non-state actors and institutions (Biermann, 2007).

Adaptiveness: The challenge of adaptiveness is associated with the requirement of 
long term sustainability, coupled with flexibility to cope with the speed of change (Biermann, 2007; Kelman and West, 2009).

Inter-organisational level:

\section{Objective determinants:}

Access to assets (financial and human resources): Financial assets include funding available to organisations undertaking disaster management whilst human resources include the skills and knowledge of staff related to disaster management (Ekstrom et al., 2012).

Leadership, management and governance structures: The quality of leadership and degree of empowerment of staff is critical for an adaptive culture (McManus et al., 2008).

Technical capacity, tools, methods and approaches: Defining the technical capacity, tools, methods and approaches included in a disaster response system is important for understanding how the system functions, monitoring/evaluation of response outcomes, and identifying gaps for future exploration and analysis, and the capacity to adapt to unforseen stresses (FAO, 2008).

Human resource for health governance and management systems, policy, finance, education, partnership, leadership: There is a need for human resources for health $(\mathrm{HRH})$ governance, leadership and partnerships for sustained $\mathrm{HRH}$ contributions to improved population health outcomes, including HRH capacities to address disasters (WHO, 2006; WHO, 2011).

\section{Subjective determinants:}

Risk Perceptions and perceived adaptation efficacy and costs: Understanding of the risks of climate change and the likely impacts on their disaster response processes (Ekstrom et al., 2012; Kuruppu et al. 2011).

Self-efficacy beliefs: The extent an individual within an organisation believes in their own ability to adapt to the impacts of climate change (Ekstrom et al, 2012; Kuruppu et al. 2011).

Silo mentality: Represents a decentralised structure, with a more individualistic approach to achieving goals, based on a limited understanding of the overall vision of the organisation (McManus et al., 2007; Biermann, 2007).

Communications and relationships: Relates to accountability. Governance that is credible, stable and inclusive must be considered to be legitimate by all stakeholders, and is held accountable for its actions and representatives by its constituencies (McManus et al., 2007; Biermann, 2007).

Strategic vision and outcome expectancy: It has been found that regardless of how well defined the purpose or vision, the operational reality and the communication of this vision can vary from one extreme to another throughout organisations (McManus et al., 
2007).

Health workforce strategic response to evolving and unmet population health and health service needs: This can be achieved through Health workforce plans and strategies that respond to population and service needs with emphasis on the most vulnerable and excluded groups (focusing on gender, equity and vulnerability), evolving health worker functions and technological advances (WHO 2011; Thompson and Zwi, 2011).

Information and knowledge: The capacity to apply current knowledge to a situation in a creative manner, assigning virtual roles, and the ability of subsets of an organisation to assume responsibilities of absent members are considered adaptive features of an organisation (McManus et al., 2008).

Elements of social practice: Practical knowledge, common understandings / norms / culture, rules, traditional knowledge/practices and material infrastructure (Strengers, 2010).

\section{Figure 2: Map of Pacific, highlighting case study countries}

Four PICs were investigated in detail: Cook Islands, Fiji, Samoa, and Vanuatu (Figure 2), selected on the basis of criteria relevant to the objectives of the study and with the assistance of the PRG. Criteria included variations of: geographical settings; policy landscapes; health workforce density; mix of countries with recent and significant climatedriven disasters; and countries that experience tropical cyclones as an example of a rapid onset disaster (excluding most Pacific atoll nations which lie near the equator - see Walsh et al., 2012). Development indicators (e.g. varied Human Development Index - see UNDP, 2011) were also used as a criterion for selection.

During May-July 2012, the research team completed over 90 interviews with high level professionals in Australia, New Zealand and in the four case study countries. Interviews were conducted with government and non-government organisations (NGOs), UN agencies, donors and regional organisations. The semi-structured interview style, developed with the guidance of the PRG, was designed to appreciate cultural differences including the need for clear questions and flexibility when interviewing people from various cultural backgrounds. Relevant organisations were identified with the assistance of the PRG, with additional organisations and individuals identified through a snow ball sampling technique (Atkinson and Flint, 2001). The organisations interviewed are provided in Box 2.

\section{Box 2: Interviewed Organisations in Australia and the Pacific}

Australian organisations interviewed:

AusAID

The Australian Council for International Development (ACFID)

The Australian Civil Military Centre

Australian Defence Force

Australian Red Cross

NGOs such as Oxfam, World Vision, Save the Children, CARE, Caritas, Plan 


and RedR
Pacific organisations interviewed:
National Disaster Management Offices
National Climate Change Offices
Ministries of Health, Environment, Finance, Foreign Affairs (among others)
National Red Cross Societies
Faith-based organisations
NGOs of both local and international origin
Donors (e.g. AusAID, New Zealand Aid Programme)
United Nations (UN) agencies
Regional Organisations (e.g. Secretariat of the Pacific Community - SPC)

General patterns and emerging issues from participants' explanations and descriptions were developed through an inductive thematic analysis (Rice and Ezzy, 2000; Charmaz, 1990). Qualitative software, NVivo, was used as a tool to organise and analyse interview transcripts and assisted the team to code for specific themes based on the conceptual framework. The research team also met regularly during the analysis phase and collaborated closely with the PRG to ensure other data sources (including field notes, personal observations, historical knowledge, individual interpretations and collective reflexivity) were incorporated into analysis. This ensured triangulation of data from multiple sources (Hansen, 2006).

\section{Limitations}

One key limitation of the research was the core research team were from Australia and therefore 'outsiders' to the Pacific. It is recognised that this will have influenced the way research was conducted and perceived. This effect was partially mitigated by the representation of Pacific Islanders on the PRG who played a critical role in informing and guiding the chosen research approach.

Interviews were conducted in English, impacting the research in three ways. First, while all PIC interviewees were proficient in English, it was their second language, and therefore affected what and how their perspectives were expressed. Second, the framing of the topic (climate change) is of foreign origins. As noted by Nunn (2009), communication about climate change is a challenge in the Pacific and local culture and religion affects the way in which the concept is interpreted. Lastly, participants at times presented contradictory views, which may be due to pride or an imperative to provide what they thought to be the 'right' answer. For instance health care professionals in Cook Islands rated their capacity as high, yet also noted the need for significant additional human and financial resources. Equally, participants sometimes noted that they had good knowledge of climate change and relevant policies, yet also noted that education on this issue was still needed.

One further limitation was several areas affecting disaster response such as traditional coping mechanisms, indigenous knowledge, and gender issues emerged, but were not covered at significant depth. Focus on these additional issues was outside the scope of this paper and further research in this area is recommended.

\section{Results and Discussion}

Across the four case study countries, results differed to some degree as to the most important key determinants of adaptive capacity. However, there were common themes 
emerging across all countries. Highlights of key country findings are provided below, followed by results answering the three research questions.

Cook Islands: Relationships and trust were found to be key to an effective and adaptable system of disaster response in the Cook Islands. With low institutional capacity due to a small population, efficiency in disaster response required the smooth flow of information between key individuals within responding agencies, and these responding agencies needed an understanding of both the formal and informal modes of operation. Strong relationships between key individuals in the DRS were observed to lead to an awareness of agents' roles, responsibilities, capacity and gaps, which in turn lead to a legitimate and credible governance structure. Incoming disaster support to the Cook Islands was reported to need greater awareness of these formal and informal modes of operation, including issues surrounding culture and traditional governance structures. Mechanisms for requesting international assistance also needed strengthening. Past events revealed some gaps in the capacity and coordination of the Cook Islands' DRS, and some steps have been taken to overcome them, for example the establishment of the nationally owned and led Disaster Trust Fund. Despite nurses playing key roles in initial assessments and response, the capacity of the health sector was also found to be constrained. Cook Islanders were, however, keen to draw on in-country capacity first and request external assistance only where gaps were evident. Leadership of key responding bodies, such as Emergency Management Cook Islands (EMCI), was observed to be crucial for future adaptive capacity. However, current capacity may be constraining the organisational ability to implement important disaster risk reduction (DRR) or response planning initiatives.

Fiji: A relatively strong, well defined DRS with clear lines of authority was found to exist in Fiji. Leadership was viewed by interviewees as being strong and effective and included all levels of society. Research participants were well aware of Fiji's vulnerability to climate change and its impacts and were motivated to prepare but were constrained in terms of human, financial, technical and material resources. The health sector was actively involved in the DRS, with nurses playing key roles in initial assessments and response.

Communication and information sharing between the broader DRS and organisations such as Ministry of Health was reported to need strengthening. This research identified examples of Australian donor funds being channelled through NGOs and specific projects due to the current political situation in Fiji. This could be contributing to the fragmented financial management that was reportedly causing confusion for some stakeholders on how and where to access finances.

Samoa: An illustration of Samoa's DRS is provided in Figure 3. Traditional and social practices played a major role in supporting affected Samoan communities after a disaster. Churches, family and social structures enhanced adaptive capacity as individuals reportedly felt supported and comforted. Inclusion of government, NGOs, churches and the community in the decision making processes (e.g. through the Disaster Advisory Committee) allowed education, training and lessons learned to be integrated into the DRS. A strain on resource capacity was evident in Samoa, in particular with regard to $\mathrm{HRH}$, financial and technical capacity. During the immediate period after the last major disaster (the 2009 earthquake and tsunami), a lack of technical capacity of front line responders exacerbated by communication issues, contributed to confusion, duplication and gaps in response. Many lessons from this event were being discussed and implemented throughout the DRS. The National Disaster Management Office (NDMO) was considered to be strong in leadership but lacked sufficient human resources.

Figure 3: Samoa's national disaster response structure (Government of Samoa, 2011)

Vanuatu: Vanuatu's NDMO provided a level of leadership that was supported by relatively high capacity and support from other organisations both in Vanuatu and from outside. The 
credibility and legitimacy of the DRS was heavily tied to the NDMO and the Vanuatu Humanitarian Team (VHT), with its multi-sectoral membership that was reported to function effectively as a result of pre-existing relationships and an internal governance arrangement (through its clusters) that was well understood. The shared perceptions of risk across Vanuatu's DRS, coupled with (or perhaps as a result of) the existence and leadership of the $\mathrm{VHT}$, had led to an overall shared strategic vision for coping with the impacts of climate change and disasters in Vanuatu. The establishment of the National Advisory Board for disaster risk reduction and climate change adaptation (NAB) provided an example of a DRS with a future focus and vision of how it would like to progress. While the health sector's perceptions of risk were aligned with those of the rest of the DRS, its limited capacity constrained its ability to fully participate in implementation of the strategic vision. Institutional and individual capacity building within the health sector was required, appreciating elements of culture, geography and lessons from the past in the development of future initiatives.

\section{Research Question 1: What constitutes the 'disaster response system' (DRS) for the immediate humanitarian needs post-disaster in Australia and each of the four case study PICs?}

The DRS in each PIC was led by a NDMO (or similar), and also included (to varying degrees) ministries such as Ministries of Health, Infrastructure, Internal Affairs, Foreign

Affairs and Finance. Red Cross Societies were active in each case study country, while nongovernment organisations were most active in disaster response in Vanuatu and Fiji. Churches were important agents for disaster response in all countries, with varying degrees of participation in national decision making. Traditional leaders also had limited participation in national level planning, however while their participation was considered important, it needed to be balanced by informed views and an appropriate level of involvement (see Lata and Nunn, 2012). The adaptive capacity of the DRS in each of the PICs was in part dependent on the degree to which DRR, preparedness and response had been prioritised by central government, and the degree to which key line ministries had incorporated disaster planning into their operations (see Turnbull (2004) for additional contributing factors). Ministries of Health in all case study PICs have various degrees of connections to the broader DRS however, while human resource and financial constraints were evident, it appeared that their overall involvement needed strengthening.

From the Australian disaster response perspective, AusAID led Australia's "whole-ofgovernment" approach to disaster response and was key to Australia's bilateral development efforts in the Pacific, both in terms of disaster response and ongoing development programming. This research found the bilateral mechanism relied heavily on the knowledge, experience and relationships of in-country AusAID staff and supported the adaptive capacity of the PIC DRS, through proactive understanding of the needs and capacity of PICs, and tailoring support accordingly. Australian Red Cross and NGOs were active in disaster response, with mechanisms existing to coordinate disaster response with AusAID through Australia Council for International Development (ACFID - the peak body for NGOs). Most NGOs had dual mandates of poverty reduction programming and disaster response when the need arises, and worked closely with in-country partners and increasingly each other, as lessons from past events were implemented to maximise the effectiveness of their support.

Regional and international coordinating mechanisms also exist to support disaster response. These include Australia's NGO Humanitarian Partnership Agreement (AusAID, 2010), FRANZ (AusAID, 2011), the Pacific Humanitarian Team and United Nations responses. While PIC interviewees could describe these mechanisms, it is recognised that in the Pacific, understanding does not always equate to endorsement or compliance. This is a complex issue related to Pacific culture, and permeates decision making and Pacific governance structures more generally (see Duncan, 2008 and Turnbull, 2004). Elements of regional coordinating mechanisms that acknowledged these cultural complexities and supported adaptive capacity included the recognition of the need to maintain relationships and 
partnerships and to provide ongoing DRR interventions as a way to minimise the impacts of a disaster. Adaptive capacity would be further enhanced if DRS organisations considered how climate change may affect their future capacity to respond, and plan accordingly.

There were some commonalities across the four case study countries regarding the DRS for the four humanitarian needs (health care, water and sanitation, psychosocial needs and food and nutrition). In all four case study countries the limited human resources for health care, both in times of disaster response and in day-to-day operations was found to affect the ability to meet health care, food and nutrition needs. The research found that policies in place to coordinate human resources for health for disaster response varied: Cook Islands and Samoa both had clear policies in place, Fiji had generic policies, however these needed to be more clearly defined for specific disasters, and Vanuatu lacked clear policies. In all countries the health workforce was found to have a strong commitment to respond to their country's needs even in the face of limited resources and often relied on donor and NGO support to fill gaps. Elements found to constrain adaptive capacity included weak coordination and registration of overseas disaster response health personnel (except in Samoa), inadequate staff welfare and compensation policies and limited $\mathrm{HRH}$ capacity even in times of "normality." Interviewees reported a chronic lack of material resources and health care infrastructure and an overall need for accessible and appropriately targeted training.

Interviewees across all four case study countries identified the gap in capacity for postdisaster psychosocial support. Some NGOs assisted with psychosocial support, however, there was no widespread systemic support available, including support for disaster responders. Of the four humanitarian needs focused on in this research, psychosocial support was given the least attention by Australian organisations. While reliance upon local NGOs and churches is culturally appropriate and acceptable in this context, both NGO and church-based interviewees acknowledged their ability was inadequate to meet all needs. Indigenous knowledge and traditional coping mechanisms for both disaster preparedness and response is acknowledged as very beneficial. However, this knowledge is being challenged and sometimes undermined in the intersection between donor assistance and traditional leadership (Walshe and Nunn, 2012; Mercer et al., 2007 and Palmer 2005). An indication of this was seen in the limited inclusion of traditional leaders in national disaster planning and response.

WASH as an immediate post-disaster humanitarian need was relatively well served by responding organisations, although long term WASH issues remained unresolved in most countries. These findings highlight that vulnerability of the health sector needs further assessment in order to manage future risks, under a changing climate (Brooks et al., 2005; Jones et al., 2010).

\section{Research Question 2: How do inter-organisational determinants serve to strengthen or reduce adaptive capacity?}

The three main inter-organisational determinants studied were architecture, agency and adaptiveness. Each are described in turn below, outlining how they affect adaptive capacity.

Architecture: The DRS 'architecture' involves the interlinked systems, networks, policies, and institutions associated with disaster response across national, regional and international scales. This research found that formal and informal networks were both effective mechanisms for information exchange and the development of relationships both in Australia and in PICs. In the context of this research, formal networks refer to those that are formed through political, diplomatic and organisational relationships, are often evidenced by formal documentation, signatories to plans, paid membership, or diplomatic memorandums of understanding. Informal networks refer to those that are self-made among its members and occur naturally amongst people or organisations with mutual interests. The informal element 
of the Pacific disaster response architecture was an essential element which supported adaptive capacity through the forging of partnerships, personal relationships and trust. For example, as a result of strong, trusting relationships, donor representatives were reportedly invited to Disaster Management meetings in Vanuatu and Samoa, despite not being members with voting rights.

Regional organisations and networks such as WHO and the Pacific Disaster Risk Management Partnership Network work to enhance adaptive capacity through training, supporting planning processes and procedures for disaster response, and assisting PICs in their national policy development efforts. Regional political and governance issues were found to constrain adaptive capacity of the DRS in some cases. For instance when relationships between DRS organisations at the national or international level were confronted by political barriers (e.g. between Australian and Fijian governments), provision of disaster relief and ongoing DRR programming became more difficult. Examples were identified where post-disaster Australian aid was channelled through an NGO rather than directly to the government. Informal mechanisms should therefore be considered equally important and viable alternatives to the delivery of humanitarian response (Brooks and Adger, 2005). It is therefore recommended that continued support be provided for regular engagement and meetings of DRS agents and networks, to maintain relationships and simplify PICs processes for requesting external assistance.

Agency draws on concepts such as power, control and decision making. The concept of 'agency' in this research goes beyond inter-governmental co-operation and includes a focus on the partnership between 'whole of government' and non-state actors and institutions. This research found that non-state actors were significant in the area of disaster response, with NGOs, churches and UN agencies wielding considerable authority in PICs. Additionally, partnerships, relationships and trust between these agents were observed to be vital in supporting the adaptive capacity of the DRS. The research found that power, control and decision making between agents in the PIC DRS needs to be inclusive and participatory to ensure outcomes (e.g. disaster response policy) were acceptable and appropriate at all levels. Some PICs had progressed further than others in including various stakeholders (e.g. traditional and church leaders) in decision making processes and inclusivity was sometimes dependent on factors such as resource capacity and leadership. The absence of trust through lack of personal relationships between key agents in some PICs resulted in barriers to effective response, hence constraining the adaptive capacity of the PIC DRS. For example, an absence of existing relationships and trust between agents was reported to be problematic for coordinating disaster assessments and act to constrain adaptive capacity of the DRS, particularly in accessing vital assessment information. In an environment where there are a growing number of agents working in PICs, sometimes in an uncoordinated manner, it is crucial for all agents to understand the importance of relationship and trust in this context.

It is recommended that recognition of the critical coordination role of NDMOs is strengthened, appropriate involvement of traditional leaders in disaster planning and response, formal involvement of churches in disaster response at national level is encouraged and structured mechanisms to capture lessons on how to improve disaster response into policy and planning are developed.

Adaptiveness, was found to be a recurring concept across the case study countries. The challenge of adaptiveness is associated with the requirement of long term sustainability, coupled with flexibility to cope with the speed of change (Biermann, 2007). Attention was given to DRR and climate change adaptation (CCA) in all case study countries, supporting the adaptive capacity of the PIC DRS through reduction of potential disaster impacts and building resilience of communities. There was ongoing support from donors and contributions from PIC national budgets for DRR and CCA interventions with a view to build 
such resilience. However the bulk of the support was donor funded, increasingly for CCA interventions. Long term dependence on aid has been described by some authors to likely result in PICs becoming reliant on external assistance rather than developing national systems of self-reliance (Levine et al., 2011). Other authors state that PICs need to begin to "own" the issue of CCA to ensure it is sustainably addressed (Nunn, 2009). Regional organisations, donors and international partners should therefore consider ways to assist PICs to build internal capacity to respond to their own needs and take ownership of CCA. Approaches should take advantage of traditional systems and knowledge, the strong sense of cooperation and resource sharing amongst in-country organisations, and make use of existing regional networks and relationships. The design of plans to incorporate known vulnerabilities and uncertainties as well as drawing on past experiences and existing relationships are adaptive approaches being used for disaster response (e.g. Government of Cook Islands, 2012). Recommendations are put forth to place greater priority into building PIC internal response capacity with an emphasis on including traditional systems and knowledge.

\section{Research Question 3: Which objective and subjective determinants are most significant in influencing the adaptive capacity of the organisations within the 'DRS'?}

Past studies of determinants of adaptive capacity often consider those that are objective and tangible including indicators such as wealth, technology, information and technical skills (Jones et al., 2010). This study also recognised intangible, or subjective, determinants, which are critical in determining the ability of individuals and organisations to adapt (Bowen et al., 2012). Table 1 displays the most influential determinants for the four case study PICs based on the analysis undertaken within this research.

\section{Table 1: Summary of most influential determinants of adaptive capacity}

Based on synthesis across the four case study countries, the overall determinants identified as most important for adaptive capacity of organisations in the Pacific region are described below, along with recommendations on how to further support adaptive capacity for disaster response. These were 'communication and relationships', 'capacity', 'leadership, management and governance structures' and 'risk perceptions with respect to climate change'.

Communications and relationships: This key determinant of adaptive capacity relates to the building of trust, informal relationships between key individuals and accountability. Supporting literature states that for governance to be considered legitimate by all stakeholders, it must be credible, stable and inclusive, and held accountable for its actions (Biermann, 2007). Effective communication pathways based on mutually respectful relationships, an understanding of Pacific culture (and to a lesser degree, language) were also identified as essential for the development of trust amongst disaster response agents in PICs. In these small island bureaucracies, trust and personal relationships between individuals were found to have a strong influence on the adaptive capacity of the DRS (as described under Agency above). One Pacific national government interviewee noted: "We need people who are good relationship managers, so a level of trust is maintained at a high level. So when an emergency happens we can sit down and make quick decisions." Adaptive capacity of organisations was found to be enhanced when individuals and organisations maintained both informal and formal relationships with key institutions and individuals both nationally and regionally, and were engaged in regional networks. Regional networks also need to ensure a coordinated approach to engaging with PICs, given the many roles and responsibilities key individuals hold in their respective countries. 
Recommendations are to improve coordination between NDMO and Ministry of Health $(\mathrm{MoH})$ in PICs, to ensure new or emerging disaster response organisations are aware of culture and thus use both formal and informal communications, and to systematise coordination of regional meetings such that they support maintenance of formal and informal networks in the disaster response sector.

Capacity: This determinant is broad, and encompasses financial, technical and human resource capacity. This key determinant was found to be highly influential in all four case study countries. Findings indicate that a country's DRS often relied on very few people with limited resources. Human resource capacity, including continuous loss of skilled workers, in PICs is well covered in the literature (see Buchan et al., 2011; Negin, 2008; Thompson and Zwi, 2011). It was notable that low institutional capacity of PIC disaster response organisations led to high levels of vulnerability. One interviewee noted: "Because of their [population] size, we are relying on individuals rather than institutions so if a strong person goes then you lose a lot." Disasters place extra demands on agencies, including the health care workforce in terms of the number of workers, skills and the tools required to meet response needs. The research revealed a need for assessment of how workforce capacity can be improved in terms of the numbers, skills, competencies, deployment and coordination required to respond to disasters. These results also remain relevant for nonclimate driven disasters (e.g. earthquake, tsunami and volcano).

It is recommended that a focus is placed on better coordination of relevant disaster response training, for continued donor support for CCA in line with risk reduction needs, and for establishment and access to national emergency funds. In addition, findings suggest that $\mathrm{MoH}$ in PICs require support to strengthen their $\mathrm{HRH}$ needs for disaster response and need to develop strategies to address the gap in psychosocial support. Finally, it is recommended that Australian and New Zealand organisations improve availability of field medical services and, in partnership with PIC organisations, provide on-going educational support for nurses in PICs who are often the first respondents to disasters.

Leadership, management and governance structures: The quality of leadership effective management and degree of empowerment of staff are critical for an adaptive culture and donors are increasingly realising that weak governance in the Pacific acts to impede development progress (Gani, 2009). This key determinant was highly influential across all case study countries. Power, control and decision making between agents responding to disasters reportedly needed to be inclusive and participatory to ensure disaster response policies, plans and procedures are appropriate and acceptable at all levels. The collective and participatory decision making processes need to be in place prior to a disaster occurring, to ensure smooth operations and clear chain of command in disaster response. Support from traditional leaders and churches was also needed for legitimate governance (lati, 2008). Some PICs (e.g. Vanuatu and Samoa) were found to be more inclusive of stakeholders in decision making and the strength of leadership of the DRS appeared to be dependent on factors such as resource capacity and quality of leadership. The research revealed that involvement and active participation of the national health services in the national and regional DRS varied. Incorporation of indigenous knowledge for disaster preparedness has also been shown to impact adaptation (Walshe and Nunn, 2012). A recommendation to strengthen involvement of $\mathrm{MoH}$ in the wider network of disaster response agencies is proposed, and also for NDMOs to improve coordination of disaster assessments by establishing clear standard operating procedures and develop and implement clear policies and plans for disaster response. Adoption of a nationally and culturally appropriate version of the Pacific Humanitarian Team (as in Vanuatu) is also recommended to increase adaptive capacity in other Pacific countries.

Risk Perceptions with respect to climate change: This key determinant relates to an organisation's understanding of the risks associated with climate change and its likely 
impacts on their disaster response processes. Having a shared perception of the changing nature of risk associated with climate change is important to improve the adaptive capacity and DRS in a given country (Ekstrom et al., 2012). This key determinant was more influential to adaptive capacity in the Cook Islands and Vanuatu. Risk perceptions surrounding climate change and disasters in some PICs (particularly Fiji and Vanuatu) were found in part to be dependent on the priorities and obligations of organisations. The need to prioritise DRR was widely acknowledged, however, following through with implementing DRR activities and extending efforts to mainstream climate change considerations varied, and was often reported to be resource dependent. Education and awareness, particularly of traditional leaders who carry considerable authority in their communities, of the need to internally adopt a future focus on DRR and CCA was needed. Incorporating capacity building into ongoing donor supported DRR and CCA programming should encourage mutual responsibility with a view to the long term sustainability of the Pacific Island disaster response mechanisms.

A final recommendation is proposed that PIC and Australian disaster response agencies and NDMOs increase their 'future' focus to incorporate changing risk and uncertainty into their strategic planning.

\section{Conclusion}

This research set out to investigate the Pacific and Australia's adaptive capacity with regard to disaster response in a climate where baseline risk is changing. Through information gathered from key stakeholders across the Australia-Pacific region, it was found that adaptive capacity of the DRS is enhanced by: strong informal communication and relationships that operate beyond formal mechanisms, appropriate participation of traditional leaders and churches in planning and disaster response, and appropriate recognition and support for the critical role NDMO's play in disaster coordination. Adaptive capacity was found to be constrained by lack of clear policies for requesting international assistance, lack of coordinated disaster assessments, limited capacity, including limited human resources for health in disaster response. A clear need for increased capacity for psychosocial support was observed, in keeping with limitations of Australian medical services to meet specific needs post-disasters. Additionally, it was found that both PIC and Australian disaster response agencies would benefit from a strengthened 'future' focus that would help them better plan for uncertainty and changing risks. Follow up on these important findings and related recommendations will serve to enhance capacity in Pacific, as well as improve Australia and overseas disaster response with a view to support PIC development of adaptive capacity. 
Notes:

1. Health systems include the organisations, departments, policies and institutional structures established by the country's government to provide health care to the population. It was found in case study countries this system is complemented by church health services, traditional health practices and NGOs. 


\section{References}

Asia Pacific Emergency Disaster Nursing Network (APEDNN) 2010, System-wide quality improvement: The foundation of emergency and disaster resilience, APEDNN Auckland, New Zealand

Atkinson, R., Flint, F., 2001, 'Accessing hidden and hard-to-reach populations: snowball research strategies', in: N. Gilbert (ed.), Social Research Update, Department of Sociology, University of Surrey, UK.

AusAID, 2010, Humanitarian Partnership Agreement Schedule 2, Australian Agency for International Development, Australian Government, Canberra.

AusAID, 2011, Humanitarian Action Policy, Australian Agency for International Development, Australian Government, Canberra.

Biermann, F. 2007, "'Earth system governance" as a crosscutting theme of global change research', Global Environmental Change 17, 326-337.

Bowen, K.J., Friel, S., Ebi K., Butler C.D., Miller, F. and. McMichael A.J., 2012, 'Governing for a healthy population: towards an understanding of how decision-making will determine our global health in a changing climate', International Journal of Environmental Research and Public Health 9, 55-72.

Brooks, N., Adger, W.N. and Kelly, P.M. 2005, 'The determinants of vulnerability and adaptive capacity at the national level and the implications for adaptation' Global Environmental Change 15, 151-163.

Buchan, J., Connell, J., and Rumsey, M. 2011, Recruiting and retaining health workers in remote areas: Pacific Island case-studies. WHO: Geneva, Switzerland

Charmaz, K. 1990, 'Discovering chronic illness: Using grounded theory', Social Science and Medicine 30, 1161-72.

Coumou, D. and Rahmstorf, S. 2012, 'A decade of weather extremes', Nature Climate Change 2, doi: 10.1038/nclimate1452

Duncan, R, 2008, 'Cultural and economic tensions in Pacific Islands' futures', International Journal of Social Economics 35 (12), 919-929

Ekstrom, M., Kuruppu, N., Wilby, R., Fowler, F., Chiew, F., Dessai, S. and Young, W., 2012, 'Examination of climate risk using a modified uncertainty matrix framework- Applications in the water sector', Global Environmental Change, $<$ http://dx.doi.org/10.1016/i.gloenvcha.2012.11.003> 
Engle, N. 2011. 'Adaptive capacity and its assessment', Global Environmental Change 21, 647-656.

Food and Agricultural Organization (FAO) 2008, Disaster risk management system analysis: a guide book, FAO, Rome.

Gani, A. 2009. Governance and foreign aid in Pacific Island countries. Journal of International Development, 21, 112-125.

Government of the Cook Islands, 2012, 'Joint national action plan for disaster risk management and climate change adaptation (JNAP) 2011-2015', Emergency Management Cook Islands, Government of the Cook Islands.

Government of Samoa, 2011, 'Samoa's National Disaster Management Plan, 2011-2014'. Government of Samoa, Apia.

Hansen, E. 2006, Successful qualitative health research: a practical introduction. Allen and Unwin, New South Wales, Australia.

Intergovernmental Panel on Climate Change (IPCC), 2001, 'Climate change 2001: impacts, adaptation and vulnerability', Contribution of Working Group II to the Third Assessment Report of the Intergovernmental Panel on Climate Change, edited by J. J. McCarthy, O. F. Canziani, N. A. Leary, D. J. Dokken and K. S. White (eds). Cambridge University Press, Cambridge, UK, and New York, USA, 2001.

Intergovernmental Panel on Climate Change (IPCC), 2012, 'Summary for Policymakers', in: Managing the Risks of Extreme Events and Disasters to Advance Climate Change Adaptation [Field, C.B., V. Barros, T.F. Stocker, D. Qin, D.J. Dokken, K.L. Ebi, M.D. Mastrandrea, K.J. Mach, G.-K. Plattner, S.K. Allen, M. Tignor, and P.M. Midgley (eds.)]. A Special Report of Working Groups I and II of the Intergovernmental Panel on Climate Change. Cambridge University Press, Cambridge, UK, and New York, NY, USA, pp. 119.2011. Managing the risks of extreme events and disasters to advance climate change adaptation: Summary for Policymakers. Special report of the Intergovernmental Panel on Climate Change.

Jones, L., Ludi, E.,Levine, S., 2010. Towards a characterisation of adaptive capacity: a framework for analysing adaptive capacity at the local level, Overseas Development Institute Background Note, December 2010.

Kelman, I. and J. West. 2009, 'Climate Change and Small Island Developing States: A Critical Review', Ecological and Environmental Anthropology 5, 1-16. 
Knutson, T.R., Mcbride, J.I., Chan, J., Emanuel, K., Holland, G., Landsea, C., Held, I., Kossin, J.P., Srivastava, A.K. and Sugi, M. 2010, 'Tropical cyclones and climate change', Nature Geoscience 3, 157-163.

Kuruppu, N. and Liverman, D. 2011, 'Mental preparation for climate adaptation: The role of cognition and culture in enhancing adaptive capacity of water management in Kiribati,' Global Environmental Change, 21, 657-669.

Lata, S. and Nunn, P.D. 2011. Misperceptions of climate-change risk as barriers to climatechange adaptation: a case study from the Rewa Delta, Fiji, Climatic Change 110, 169-186.

Levine, S., Ludi, E. and Jones, L., 2011. Rethinking Support for Adaptive Capacity to Climate Change: The Role of Development Interventions. Overseas Development Institute, UK.

McManus, S., Seville, E., Brunsdon, D. and Vargo, J. 2007, 'Resilience management: A framework for assessing and improving the resilience of organisations', Resilient Organisations Programme, New Zealand.

McManus, S., Seville, E., Vargo, J. and Brunsdon, D. 2008, 'Facilitated process for improving organizational resilience', Natural Hazards Review May 2008, 81-90.

McMichael, A., Campbell-Lendrum, C., Corvalán, K.L. Ebi, A. Githeko, J.D. Scheraga and A. Woodward, 2003. eds. Climate change and human health: Risks and responses, WHO Geneva, Switzerland.

Mercer, J., Dominey-Howes, D., Kelman, I., and Lloyd, K. 2007, The potential for combining indigenous and western knowledge in reducing vulnerability to environmental hazards in small island developing states. Environmental Hazards 7(4), 245-256.

Negin, J. 2008, Australia and New Zealand's contribution to Pacific Island health worker brain drain. Australian and New Zealand Journal of Public Health 32(6), 507-511.

Nunn, P.D. 2009. Responding to the challenges of climate change in the Pacific Islands: management and technological imperatives, Climate Research 40, 211-231.

Palmer, I., 2005, 'ABC of conflict and disaster: Psychological aspects of providing medical humanitarian aid,' British Medical Journal 331 (7509), 152

Rice, P. and Ezzy, D. 2000, Qualitative Research Methods: A Health Focus. South Melbourne, VIC, Oxford University Press. 
Strengers, Y. 2010, 'Conceptualising everyday practices: composition, reproduction and change', Working Paper No. 6. Centre for Design, RMIT and University of South Australia.

Thompson, L. and Zwi, A. 2011. 'HRH in Public Health Emergencies in developing countries: an overview', October 2011, Human resources for Health Knowledge Hub. University of New South Wales, Australia.

Turnbull, J. 2004, Explaining complexities of environmental management in developing countries: lessons from the Fiji Islands. The Geographical Journal, 170(1), 64-77.

UNDP 2011, Human Development Report 2011, viewed 12 December 2012, <http://hdr.undp.org/en/media/HDR 2011 EN Complete.pdf>

Walker, B., Holling, C.S., Carpenter, S.R., Kinzig, A., 2004, 'Resilience, adaptability and transformability in social-ecological systems', Ecology and Society 9 (2), 5.

Walsh, J.E., Mclnnes, K.L. and McBride, J.L. 2012, Climate change impacts on tropical cyclones and extreme sea levels in the South Pacific - A regional assessment. Global and Planetary Change, 80, 149-164

Walshe, R. A., \& Nunn, P. D. 2012, Integration of indigenous knowledge and disaster risk reduction: A case study from Baie Martelli, Pentecost Island, Vanuatu. International Journal of Disaster Risk Science, 3(4), 185-194.

World Bank, 2010, 'World Development Report 2010: Development and Climate Change', World Bank, Washington DC, USA.

World Health Organization (WHO) 2006, 'The World Health Report 2006 - working together for health', Geneva, Switzerland: WHO.

World Health Organization (WHO) 2011, 'Human resources for health action framework: For the Western Pacific Region (2011 - 2015)' Draft. Manila, Philippines: WHO WPRO. 
Table 1: Summary of most influential determinants of adaptive capacity

\begin{tabular}{|c|c|c|c|}
\hline Cook Islands & Fiji & Samoa & Vanuatu \\
\hline $\begin{array}{l}\text { 1. Communications } \\
\text { and relationships, } \\
\text { information and } \\
\text { knowledge } \\
\text { 2. Leadership, } \\
\text { management and } \\
\text { governance } \\
\text { 3. Capacity (human } \\
\text { resources, financial } \\
\text { and technical) } \\
\text { 4. Risk Perceptions }\end{array}$ & $\begin{array}{l}\text { 1. Information and } \\
\text { knowledge } \\
\text { 2. Risk Perceptions } \\
\text { 3. Communications } \\
\text { and relationships } \\
\text { 4. Financial } \\
\text { Capacity } \\
\text { 5. Leadership, } \\
\text { management and } \\
\text { governance }\end{array}$ & $\begin{array}{l}\text { 1. Traditional and } \\
\text { social practices } \\
\text { 2. Capacity } \\
\text { (human resources, } \\
\text { financial and } \\
\text { technical) } \\
\text { 3. Leadership, } \\
\text { management and } \\
\text { governance } \\
\text { 4. Tools, methods } \\
\text { and approaches }\end{array}$ & $\begin{array}{l}\text { 1. Communications } \\
\text { and relationships, } \\
\text { information and } \\
\text { knowledge } \\
\text { 2. Leadership, } \\
\text { management and } \\
\text { governance } \\
\text { 3. Capacity (human } \\
\text { resources, financial } \\
\text { and technical) } \\
\text { 4. Risk Perceptions } \\
\text { 5. Strategic vision } \\
\text { and outcome } \\
\text { expectancy }\end{array}$ \\
\hline
\end{tabular}

